\title{
Morphology and Phase Ordering in Polymer-Derived Nanoceramics
}

\author{
S.T. Taylor, J. Wan, P.R.L. Malenfant, A. Alizadeh and M. Manoharan \\ GE Global Research, One Research Circle, Niskayuna, NY 12309
}

A major effort in nanotechnology research is to develop novel processing schemes for the nanoscale assembly of materials with long-range order. Bottom-up approaches to materials processing make use of various structure-directing agents to engineer microstructure in a controlled fashion, enabling the fabrication of unique bioinspired structures that feature chemical or phase ordering at nanometer length scales. The realization of ordered and/or hierarchical microstructures has long been the goal of materials scientists trying to mimic the morphology and unique mechanical properties of natural composites such as conch, nacre, dentin and bone [1]. Here, we describe progress towards the rational design of bulk multiphase, polymer-derived ceramics with nanoscale order, using imaging and spectroscopy techniques in the TEM to characterize various aspects of microstructure evolution.

Our approach utilizes self-assembly of block copolymers to dictate nanoscale structure, while phase chemistry is controlled via the incorporation of one or more ceramic precursors [2]. Various morphologies ranging from lamellar to cylindrical to other ordered architectures are possible (see Fig. 1), depending on the nature of block copolymer and precursor chemistry and loading. Electron energy-loss spectroscopy (EELS) and energy-filtered transmission electron microscopy (EF-TEM) are used to characterize specific phase-targeting of ceramic precursors with nanoscale resolution. Fig. 2 shows a STEM dark-field image and associated EELS chemical line profile acquired from a lamellar structure containing silicon carbonitride ceramic precursor. Clearly, the Si signal stemming from the SiCN precursor is confined to the bright lamella, indicating successful phase targeting of ceramic precursor in the as-assembled structure. Successful phase targeting of a boron carbonitride precursor is demonstrated in the energy-filtered images of Fig. 3.

After self-assembly of the block-copolymer/precursor mixture, the material is pyrolyzed at elevated temperature to form a multiphase ceramic, with the goal of retaining long-range chemical and phase ordering. Figure 4 shows energy-filtered and phase-contrast images acquired from a pyrolyzed material where boron nitride precursor has been introduced to one of the block copolymer phases. These images demonstrate that an ordered inverse cylindrical morphology has been retained (Fig. 4a), and that BN chemistry has been preserved (Fig 4b), following pyrolysis at $1000 \mathrm{C}$. The HRTEM image in Fig. 4c reveals the turbostratic character of the pyrolyzed BN material.

Used in concert, spatially-resolved imaging and spectroscopy techniques in the TEM enable detailed studies of structure and phase evolution in polymer-derived nanostructured ceramics, and provide the only direct means for evaluating structure and chemistry simultaneously in these unique nanophase materials.

\section{References}

[1] S. Kamat et al., Nature 405 (2000) 1036.

[2] J. Wan et al, Chem. Mat. 17 (2005) 5613. 

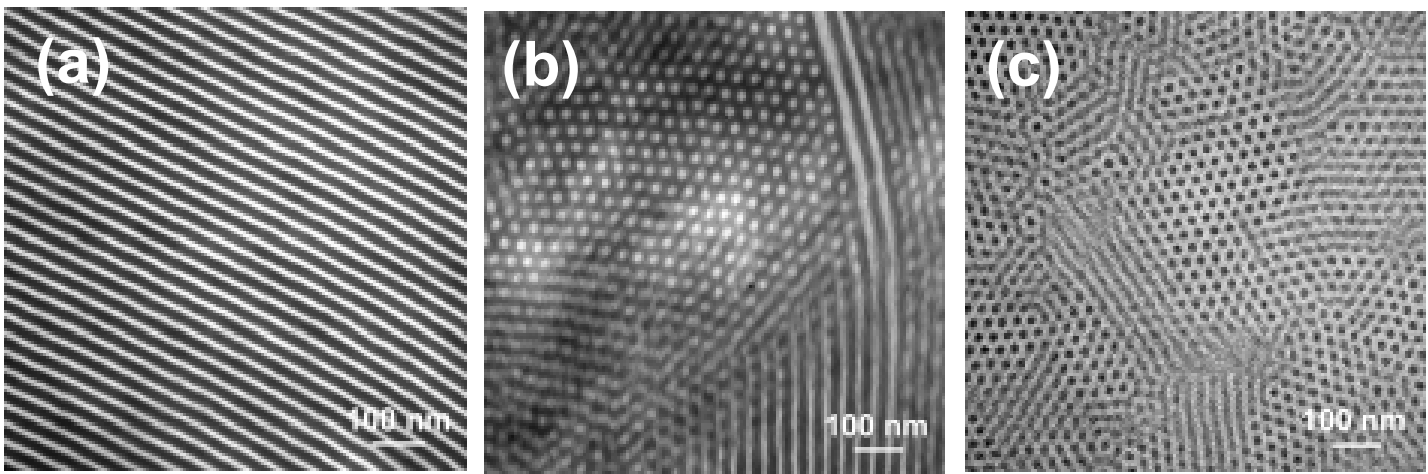

Fig. 1. (a) Lamellar, (b) cylindrical and (c) inverse cylindrical morphologies in selfassembled block-copolymers containing phase-targeted ceramic precursors.

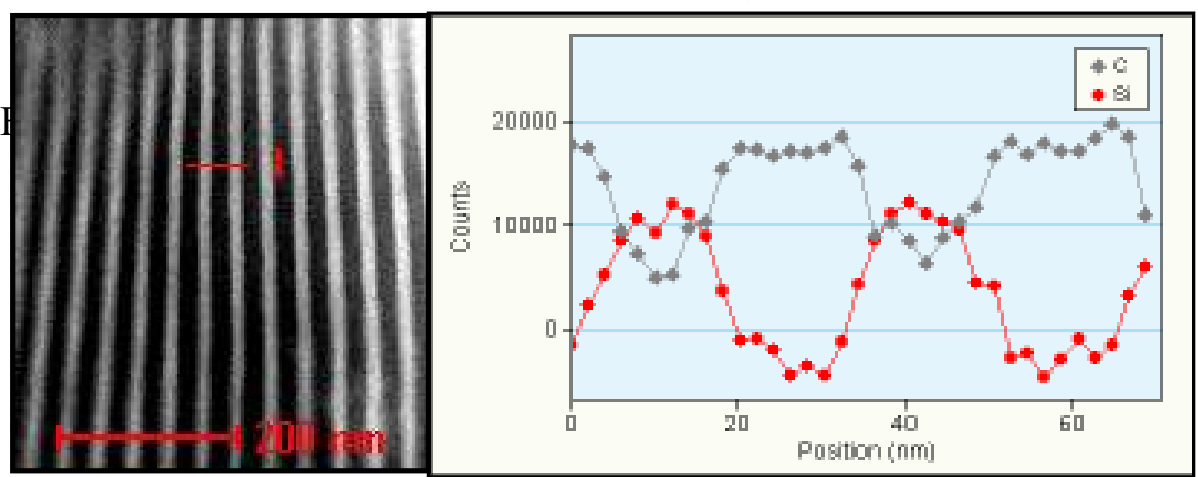

Fig. 2. EELS line profile across lamellae reveals block-specific phase targeting of SiCN precursor.
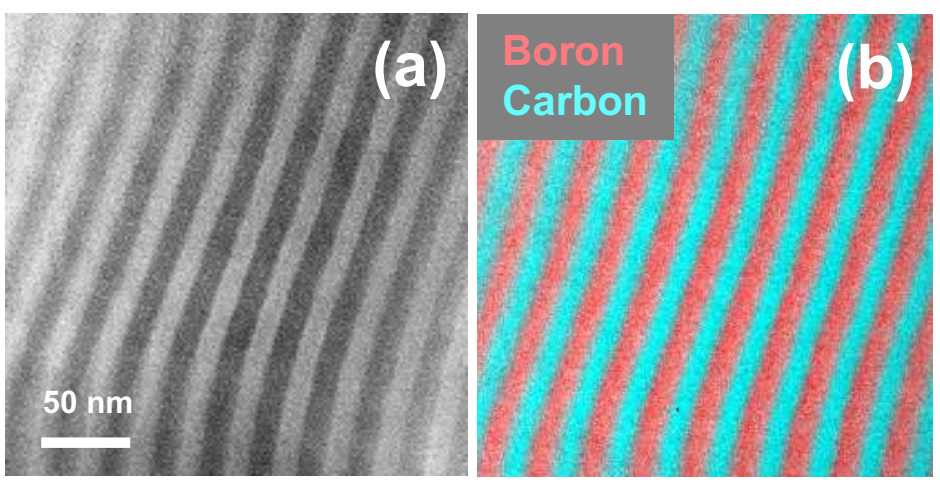

Fig. 3. (a) Bright field image and (b) elemental map of block copolymer sample containing boron carbonitride precursor (mapped in red) in one of the block phases.
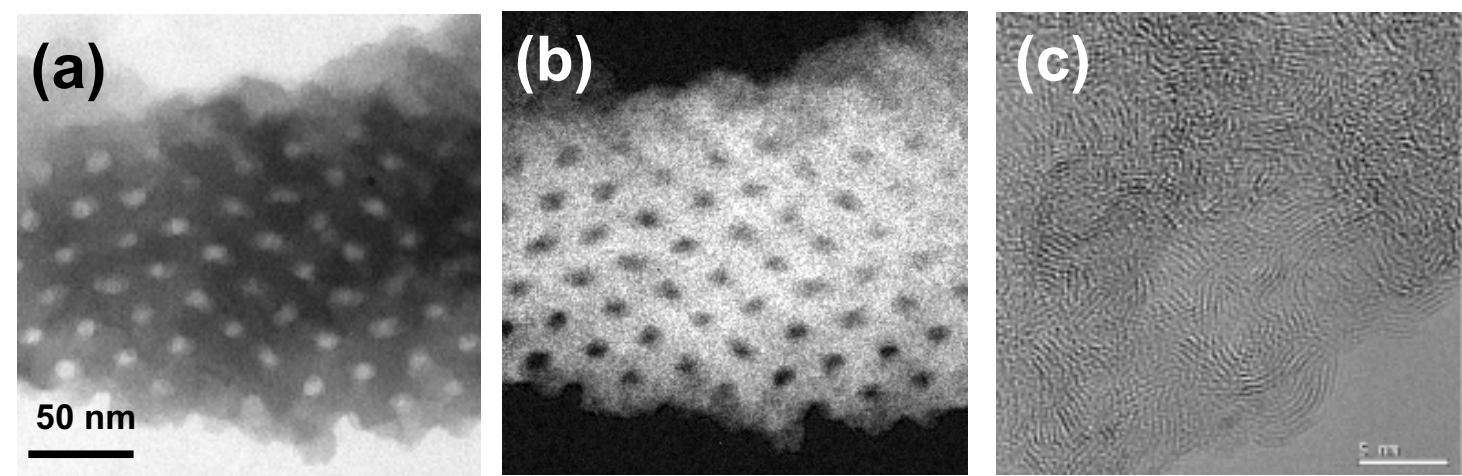

Fig. 4. (a) Bright field image, (b) elemental boron map and (c) HRTEM image of pyrolyzed block-copolymer sample containing boron nitride precursor. 